\title{
PC based Characterization of Optoelectronic Devices with Reference to Engineering Applications
}

\author{
Suresh V. Bhagwat. \\ Associate Professor, Department of Electronic Science, \\ S.N. Arts, D.J.M. Commerce \& B.N. Sarda Science College Sangamner-422605, Dist.Ahmednagar,M.S.,India.
}

\begin{abstract}
This Paper reports a PC based Characterization setup and parameter measurement of Optoelectronic Devices with reference to Engineering Applications. The setup builds up more than forty test experiments for LED, LDR, Photodiode, Phototransistor, Optocouplers, Optoisolators and Optical fibers. Emphasis is given on Testing the Electrical, spectral and spatial characteristics of these devices. Further simple tests can also be carried out for Optoelectronic systems such as Analog and digital communication links, voice communication links etc. The Design of the setup is made flexible so that the experiments can be arranged in modular form. The modules include a PC add-on card for Analog to digital and Digital to Analog conversion. Voltage to current and current to voltage conversion, Stepper motor driver and position sensing unit supported by appropriate driver software. The simplest job of user is only to connect this module at appropriate position and run the software re-quire to run the experiment. The user friendly software offers the facility of selection of the experiment(s) and the results are displayed in graphical and tabular forms.
\end{abstract}

\section{General Terms}

Optoelectronics Devices, modular Experiments.

\section{Keywords}

PC Based characterization, PC Based Experiment.

\section{INTRODUCTION}

Optoelectronics is recently developing branch of Electronics[1]. This growth of Optoelectronics has a great impact on social and scientific development. Number of applications such as optical fiber communication[2], optical remote control devices, optical computing and so on have been developed during recent times. The subject has thus become an important update in the undergraduate and postgraduate curriculum of science and engineering faculty. Basic understanding of the working of components, systems and developing skills for measurement of parameters has become an important step in learning modern Optoelectronics.

In this paper a module has been described which is developed to perform the testing of Optoelectronic components and systems automatically. The set up builds up more than forty test experiments for LED, LDR, Photodiode, Phototransistor, Optocouplers, Optoisolators and Optical fibers. Emphasis is given on testing the electrical, optoelectrical, spectral, and spatial characteristics of these devices. Further, simple tests can also be carried out for optoelectronic systems such as analog and digital communication links[3], voice communication links etc. The following section describes the design details of the modular kit

\section{DESIGN DETAILS}

For the sake of simplicity in design and working the experiments have been grouped into basic component characterization and system characterizations and accordingly the modules are proposed.

Each of the module is further subdivided in to two major parts, which is the base unit and the individual experiment modules. The base unit consists of blocks commonly required for different experiments such as power supply lines, Data acquisition system, Parallel DIO etc. The user has to connect the individual experiment module to the 26 pin FRC provided on the front panel of the base module and select the appropriate software to perform the experiment[3]. Following section describes the constructional details of Base unit.

\subsection{Base Unit}

This unit integrates the common requirements of all experiments. This includes power supply, printer port control lines and data acquisition system. All these lines are combined together to form a single 26 pin FRC. The user can connect this FRC to different modules ( one at a time ) to perform the experiment. Fig. 1 shows the schematic of the base unit.

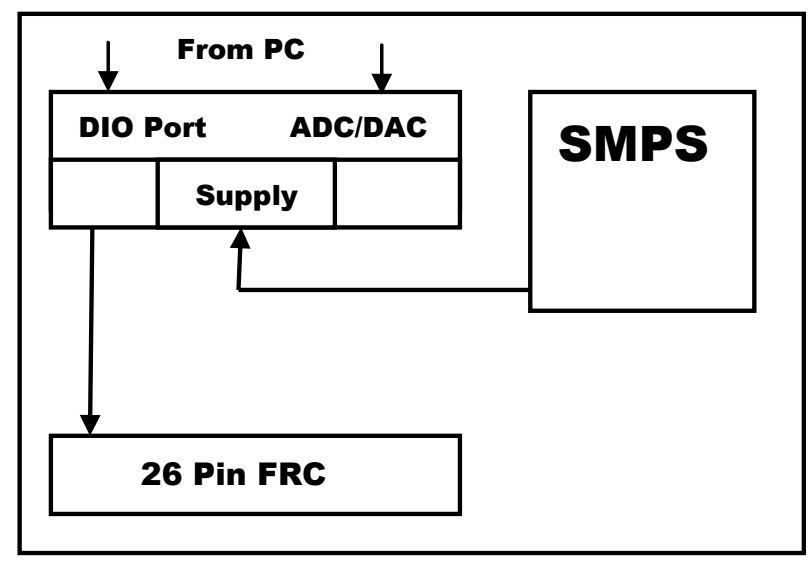

Fig.1 Block Diagram of Base Unit

As there are about 15 modules in the kit it would be inadequate to describe each one of them in details. This would require lot of space and would have many repetitions also. It is therefore plan to explain details of one module design for LED characterization. Following section deals with this module in details.

\subsection{LED Module}

A specially designed gear system is coupled to stepper motor axis, on the top of which a LED holder holds the device exactly at the centre. The stepper motor is connected to printer 
port through a front of LED a photo-detector is mounted at a fix distance. A standard $\mathrm{AD} / \mathrm{DA}$ card is used for data acquisition which has a DAC channel and 8 ADC channels. A programmable current is passed through LED through $\mathrm{V}$ to I convertor connected to DAC of data acquisition card. The voltage developed across $\mathrm{LED}$ is acquired by $\mathrm{ADC}$ channel \#1. Similarly the voltage developed across the photodiode ( due to variation of LED intensity) is acquired through ADC channel \#2. The schematic block diagram of the system is as shown in fig 2.

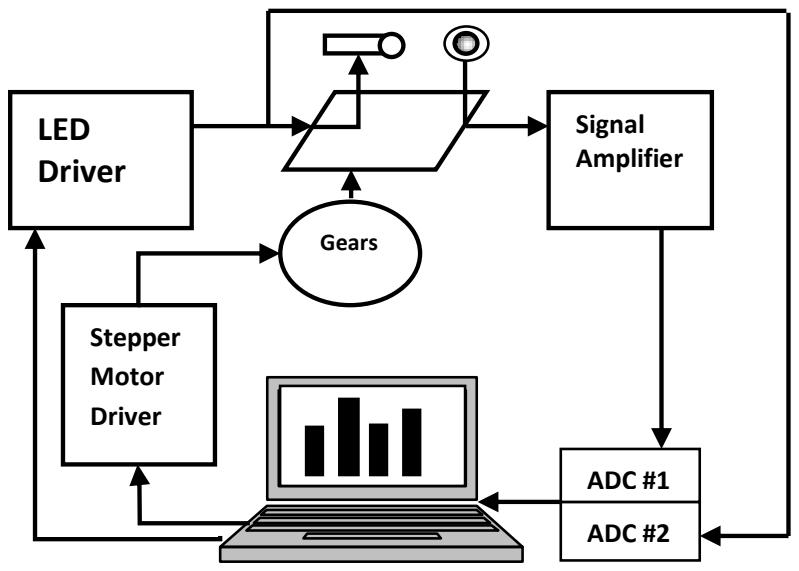

Fig.2 Block Diagram of source module.

\subsection{Mechanical Design}

A stable and a accurate mechanical system is always desired to get good results. A mechanical system used here serves two main purposes, one is to provide a stable platform for LED and Photodiode, secondly to sense the angle in terms of voltage. As shown in fig. 3, the present system consists of two gears. The first one is coupled to stepper motor and the other one to potentiometer. A LED holder is mounted on the top of first gear and in front of LED at a particular distance photodiode is placed. The conversion ratio of gear is 6:1 i.e. when the first gear rotates 6 times the second completes only one rotation. This improves the accuracy in angle measurement. A potential divider arrangement used here provides a voltage which is proportional to angle. Thus a mechanical system provides rotation of LED and gives angle in terms of voltage. This is further given to PC through Analog to Digital Convertor.

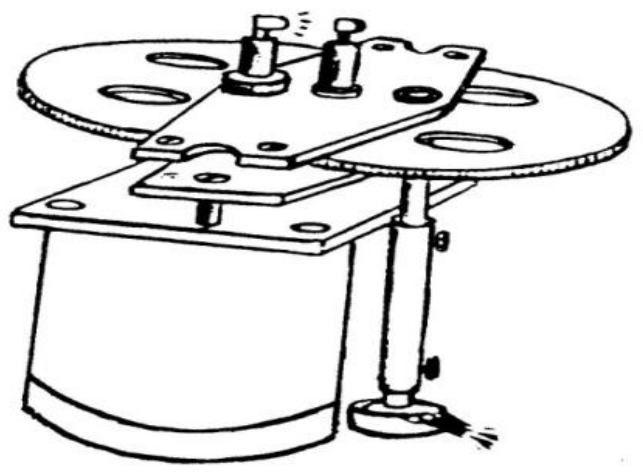

Fig.3 Mechanical System

\subsection{Design of $\mathbf{V}$ to I Convertor}

To pass programmable current through LED a V to I convertor[4] is necessary at input end while to acquire voltage across LED an ADC is required at output end. Therefore a circuit diagram for $\mathrm{V}$ to I convertor is as shown in fig. 4.

Vled $=$ Vout - Vin

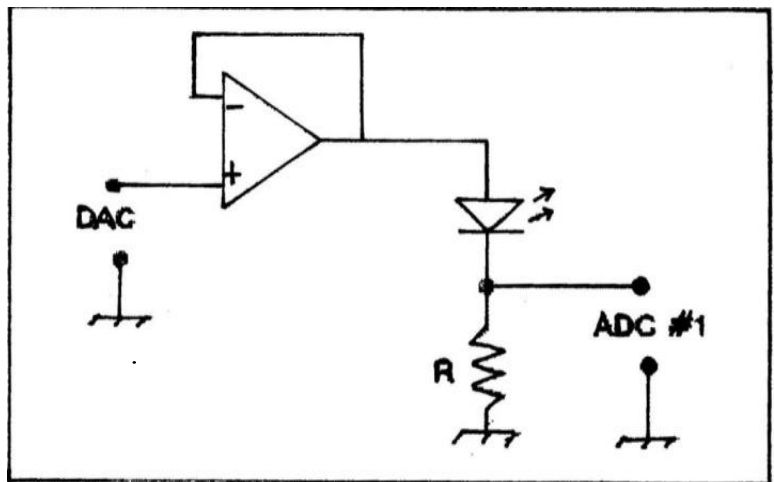

Fig. $4 \mathrm{~V}$ to I Convertor

It simply consists of a Opamp as a non-inverting unity gain buffer. The LED is connected at the output through a current limiting resistor ' $R$ '

The Voltage developed across LED is, Vled $=$ Vout - Vin $\ldots \ldots \ldots \ldots \ldots \ldots \ldots(1)$

Similarly the current through LED is,

$$
\text { Iled }=\text { Vled } \div \text { R. }
$$

\subsection{Design of Amplifier}

The current variation of photodiode is in the range of microamperes, this current variation is converted into voltage by the voltage divider arrangement. But the voltage developed is very small, therefore an amplifier needed. The circuit is as shown in fig. 5. As seen, it is basically a combination of noninverting buffer and an inverting amplifier.

Amplification factor is nothing but gain of amplifier which in present case is given by

$$
\text { Gain }=-R f \div R i
$$

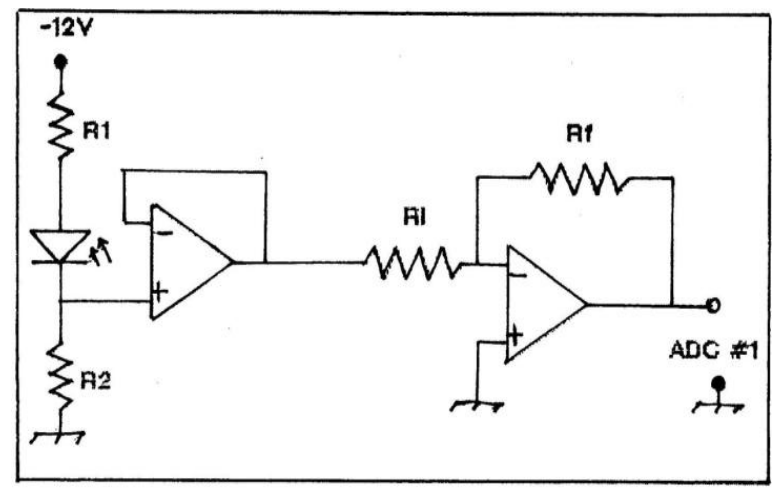

Fig. 5 Signal Amplifier

So that by adjusting $\mathrm{Rf}$ and $\mathrm{Ri}$ the required gain can be obtained and the amplified signal is then given to ADC for further processing.

\subsection{Stepper Motor Driver Card}

It consists of a non inverting buffer and a coil driver as shown in fig.6. The computer provides the required electrical pulses through the printer port. These pulses are then applied to coil driver which boosts the current .When these pulses along with 
supply voltage applied to steeper motor, the motor rotates[4] The speed of rotation is governed by the rate at which the pulses are applied[5]

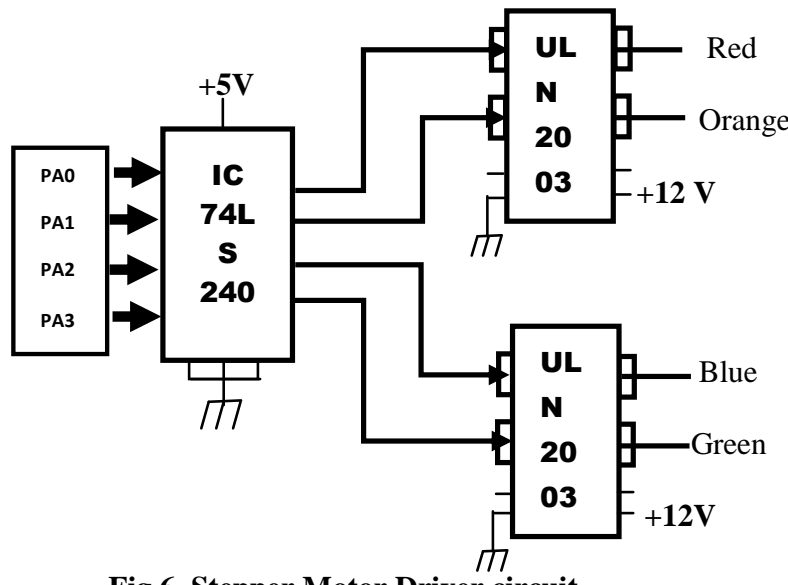

Fig.6 Stepper Motor Driver circuit

\section{DRIVER SOFTWARE}

Conventionally, an experiment is planned and carried out manually. The observations are tabulated and then interpreted. This is a time consuming process and also can leads to several errors. With the introduction of PC the planning, adjustments, data acquisition, analysis and presentation becomes very easy and fast.

The operation of the present module is carried out under program control[6]. The only job of user is to connect this module to the base unit at an appropriate position and run the required software. The software consist of three major parts,

1. Panel of the experiment.

2. Experiment control and data acquisition.

3. Parameter analysis.

\section{Panel of experiment.}

The front panel of this experiment is designed[7] in such way that user can have flexibility of selecting different options. There are six options on the right side of the screen which includes spatial characteristics, I-V characteristics, Current Intensity relation, Parameter evaluation, Representation of results and Experiment over. Below these options a window is opened in which user has to enter his option.

\section{Experiment control and data acquisition.}

The experiment is fully PC controlled[8]. As said above to perform the experiment, user has to only select the option. There are five option, the description of which is given below.

\section{Option 1 :}

Selection of this option will transfer the control to experiment no.1.i. e. 'Spatial characteristics of LED'. This will load the corresponding files in the memory. The software here performs three major activities, first is to send an appropriate voltage to $\mathrm{V}$ to I convertor, secondly it provides the data pulses to stepper motor through the printer port and thirdly to acquire the data when LED is rotating on the axis. After the program execution the LED is initially off and will get aligned to either -90 or +90 degree which is achieved with the help of a potentiometer attached as an angle sensor. After the alignment is over the LED is made on by passing a suitable current through it. Then LED starts rotating at an angle of 1.8 degree and each time the data is acquired. This data acquisition will stop when LED completes 180 degree rotation. This data is now ready for analyzing parameter using option 4. By changing the LED the experiment can be repeated several times.
Option 2:

When option 2 is selected the control will be transferred to experiment no. 2 i.e. I-V characteristics of LED. Here the current through LED is varied by varying the DAC voltage in small increments from 0 to $5 \mathrm{v}$. During each step the voltage drop across LED is measured and on line I-V characteristics is plotted on the computer screen. Similarly the experiment can be repeated for several LEDs. The data file stores the result in two column format. The first column represents current and second represents voltage across LED. Again this data file can be used for offline inspection and analysis of experiment results.

Option 3.

This will select third experiment i.e. 'Current Intensity relation'. Here again the current through LED is varied in suitable steps and the voltage developed across photodiode is measured. In this experiment, LED and photodiode are infront of each other. Again the data is stored in file and the relation is established.

\section{Option 4:}

This is for parameter evaluation of LED. Before selecting this option the user should see that at least one of the above experiment is performed. This option plays with the data stored in the files which is an outcome of an experiment. This can plot Polar graph, I-V characteristics, current intensity relation and can also find different parameters associated with each experiment.

\section{Option 5:}

This option is used for representation of results in a tabular form, giving various parameters, applications, comparisons of different LED.

\section{Option 6:}

This is just used to quit from the program. A typical panel while data acquisition[9]is as shown in fig. 7.0.

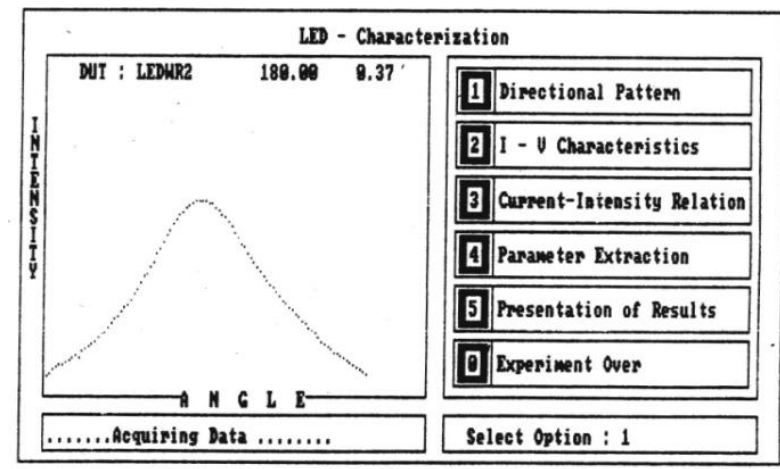

Fig.7 Panel of the Experiment

\section{RESULTS}

Selection of option 1 will perform experiment no. 1. i.e. Spatial characteristics of LED. Figure 8 shows one such polar plot.

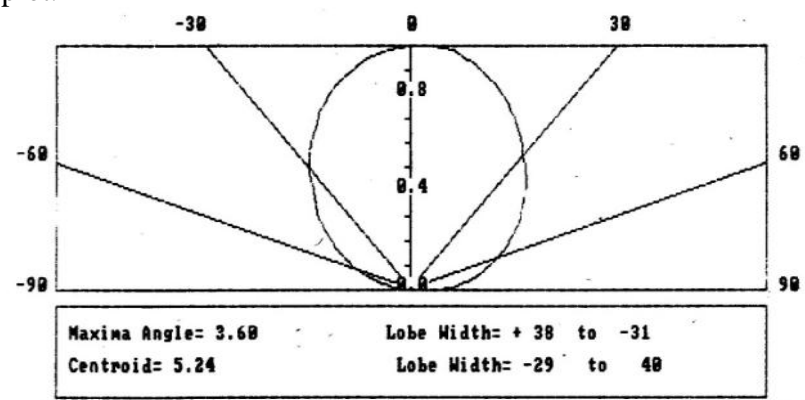

Fig.8 Polar response of Typical LED 
The program rotates LED through 180 degree and while rotating, the data is acquired through ADC channel. The online plot of this data is shown on the left side of the Computer screen. After the data acquisition, it gives the polar response of the corresponding LED. The same experiment is repeated for different LEDs of different colors, different shapes and size. From the nature of graph it is decided that which LED is best suitable for optical fiber communication. One such LED not suitable for this communication is as shown in fig 9.

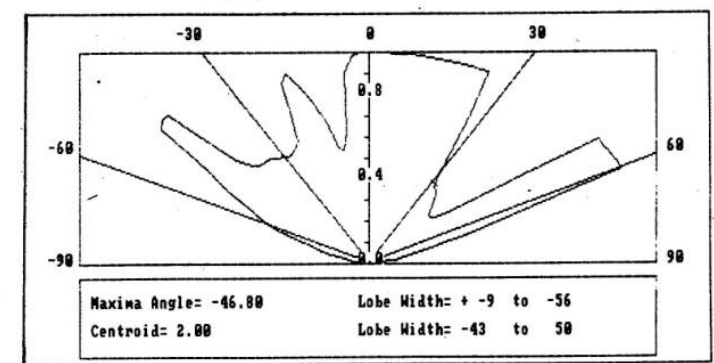

Fig 9 Typical plot of LED

The software also allows to see 4 overlapping plots at a time which can be used for comparison of different LEDs. One such plot for four green LEDs is as shown in figure 9.

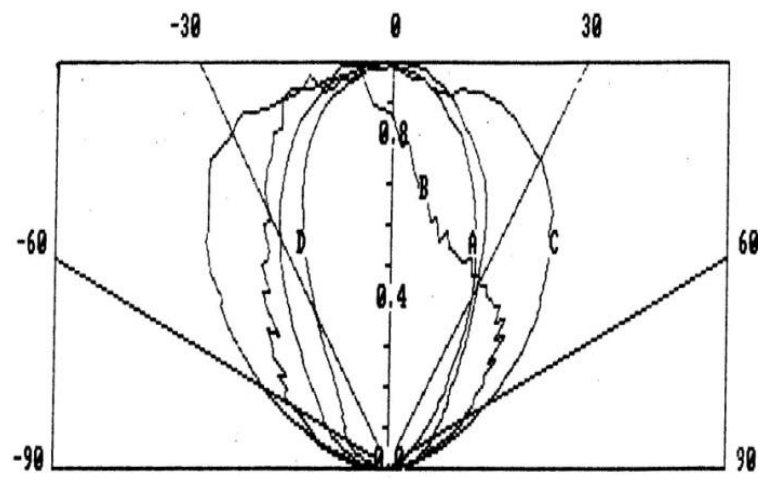

Fig.10 Polar response of 4 different LEDs.

Option2 will give online I-V characteristics of a LED. A typical I-V plot is as shown in figure 10 . Similarly option3 will show the current intensity relation. The tabulated results shown in Table 1 is the output of option4. It shows different parameters of LED and their comparison.

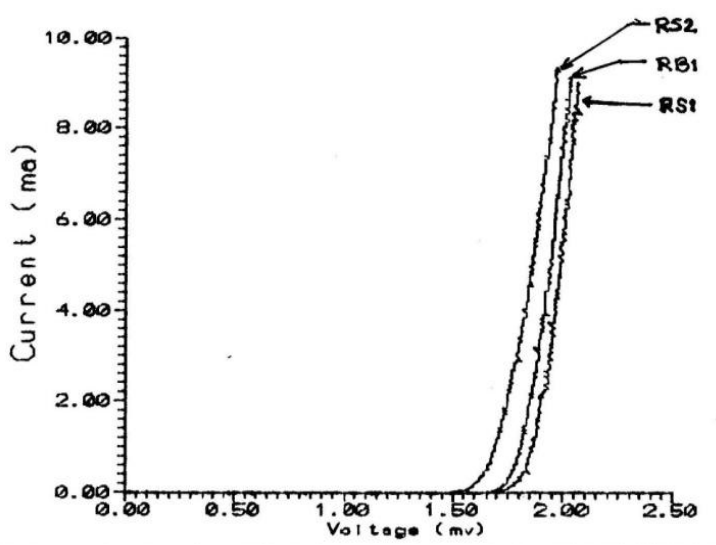

Fig. 11 V-I Characteristics of 3 different LEDs.
TABLE 1

\begin{tabular}{|l|c|c|c|}
\hline LED & Max Angle & Lobe Width & Vth \\
\hline GS1 & -1.8 & 29 to -34 & 1.80 \\
\hline GB1 & -14.0 & 27 To -41 & 1.80 \\
\hline GF1 & -9 & 52 to -56 & 1.84 \\
\hline GFR1 & 1.8 & 31 to -25 & 1.80 \\
\hline
\end{tabular}

\section{CONCLUSIONS}

As this module is proposed as a learning tool, some feedback tests are carried out before performing the actual experiment. If the user is a novice then the software also provides basic background about LED. Thus the module helps in learning and measuring the different parameters of LED. Similar modules are developed for various Optoelectronic Components like LDR, photodiode, optocouplers etc. The other module helps in analyzing systems. It is hoped that these modules will be more useful to undergraduate and postgraduate students as well as for the scientists working in Optoelectronics field.

\section{ACKNOWLEDGMENTS}

Author wish to acknowledge the authorities of Sangamner College. Sangamner and Dept. of Electronic Science, University of Pune.

\section{REFERENCES}

[1] Morris Tischler, 'Optoelectronics, Fiber optics and Lasers', Atext lab manual, second edition, Mc Graw-Hill Publication.

[2] Gerd Kaiser,"Optical Fiber Communications",second edition,Mc-Graw Hill ,INC,Newyork,1991

[3] A.K.Ghatak and M.R.Shenoy,"Fiber Optics through experiments",Viva, New Delhi 1994.

[4] Zbar,Malvino and Miller "Basic Electronics",A Text Lab manual, Seventh Edition,Tata Mc-Graw Hill Edition 1999.

[5] S.V. Bhagwat and A.D. Shaligram, "Stepper motor : basics to applications",internal report.

[6] "Turbo Pascal", The ultimate Pascal environment manual version 3.0

[7] C.M. Pandit,"Advance Techniques in Turbo Pascal”,BPB Publications,First Edition, New Delhi.

[8] Graphical Kernel systems (GKS)'Information processing in Computer Graphics", ACM transactions in Graphics, February 1984.

[9] B. Govindrajalu,"IBM PC and Clones",Tata MC Graw Hill, 1993. 